\title{
Patients with type A acute aortic dissection presenting with major brain injury: Should we operate on them?
}

\author{
Marco Di Eusanio, MD, PhD, ${ }^{a}$ Himanshu J. Patel, MD, ${ }^{\mathrm{b}}$ Christoph A. Nienaber, MD, FACC, FESC, ${ }^{\mathrm{c}}$ \\ Daniel M. Montgomery, BS, ${ }^{b}$ Amit Korach, MD ${ }^{\mathrm{d}}$ Thoralf M. Sundt, MD, ${ }^{\mathrm{e}}$ Carlo DeVincentiis, MD ${ }^{\mathrm{f}}$ \\ Matthias Voehringer, MD, ${ }^{\mathrm{g}}$ Mark D. Peterson, MD, PhD, ${ }^{\mathrm{h}}$ Truls Myrmel, MD, PhD, ${ }^{\mathrm{i}}$ \\ Gianluca Folesani, MD, ${ }^{a}$ Magnus Larsen, MD ${ }^{i}$ Nimesh D. Desai, MD, ${ }^{\mathrm{j}}$ Joseph E. Bavaria, MD, ${ }^{\mathrm{j}}$ \\ Jehangir J. Appoo, MD, ${ }^{\mathrm{k}}$ Teresa M. Kieser, MD, ${ }^{\mathrm{k}}$ Rossella Fattori, MD, ${ }^{\mathrm{a}}$ Kim Eagle, MD, ${ }^{\mathrm{b}}$ \\ Roberto Di Bartolomeo, MD, ${ }^{\mathrm{a}}$ and Santi Trimarchi, $\mathrm{MD}^{\mathrm{f}}$
}

Objectives: The management strategy remains controversial for patients presenting with type A acute aortic dissection with cerebrovascular accident or coma. The present study aimed to help guide surgeons treating these high-risk patients.

Methods: Of 1873 patients with type A acute aortic dissection enrolled in the International Registry for Acute Dissection, $87(4.7 \%)$ presented with cerebrovascular accident and $54(2.9 \%)$ with coma. The hospital and 5 -year results were stratified by the presence and type of brain injury (no injury vs stroke vs coma) and management type (medical vs surgical). Independent predictors of short- and mid-term survival were identified.

\begin{abstract}
Results: Presentation with shock, hypotension, or tamponade $(46.8 \%$ vs $25.2 \% ; P<.001)$ and arch vessel involvement $(55.0 \%$ vs $36.1 \% ; P<.001)$ was more likely in patients with brain injury. Surgical management was avoided more often in patients with coma $(33.3 \%)$ or cerebrovascular accident $(24.1 \%)$ than in those without brain injury $(11.1 \% ; P<.001)$. The overall hospital mortality was $22.7 \%$ without brain injury, $40.2 \%$ with cerebrovascular accident, and $63.0 \%$ with coma $(P<.001)$. Mortality varied among the management types for both cerebrovascular accident $(76.2 \%$ medical vs $27.0 \%$ surgical; $P<.001)$ and coma $(100 \%$ medical vs $44.4 \%$ surgical; $P<.001)$. Postoperatively, cerebrovascular accident and coma resolved in $84.3 \%$ and $78.8 \%$ of cases, respectively. On logistic regression analysis, surgery was protective against mortality in patients presenting with brain injury (odds ratio $0.058 ; P<.001)$. The 5 -year survival of patients presenting with cerebrovascular accident and coma was $23.8 \%$ and $0 \%$ after medical management versus $67.1 \%$ and $57.1 \%$ after surgery (log rank, $P<.001$ ), respectively.
\end{abstract}

Conclusions: Brain injury at presentation adversely affects hospital survival of patients with type A acute aortic dissection. In the present observational study, the patients selected to undergo surgery demonstrated improved late survival and frequent reversal of neurologic deficits. (J Thorac Cardiovasc Surg 2013;145:S213-21)

Supplemental material is available online.

Type A acute aortic dissection (TA-AAD) is a lethal condition requiring emergency surgery. ${ }^{1,2}$ However, in patients with TA-AAD presenting with major brain injury, surgical

From the Department of Cardiac Surgery, ${ }^{\text {a }}$ S. Orsola-Malpighi Hospital, Bologna, Italy; Department of Cardiac Surgery, ${ }^{\mathrm{b}}$ University of Michigan, Ann Arbor, Mich; Department of Internal Medicine, ${ }^{\mathrm{c}}$ University Hospital Eppendorf-Rostock, Rostock, Germany; Department of Cardiothoracic Surgery, ${ }^{\mathrm{d}}$ Hadassah Hebrew University Medical Center, Jerusalem, Israel; Thoracic Aortic Center, Massachusetts General Hospital, Boston, Mass; Thoracic Aortic Research Center, ${ }^{\mathrm{f}}$ I.R.C.C.S. Policlinico San Donato, San Donato, Italy; Department of Cardiology, ${ }^{\mathrm{g}}$ Robert-Bosch Krankenhaus, Stuttgart, Germany; Division of Cardiac Surgery, ${ }^{\text {h }}$ St. Michael's Hospital, Toronto, Ontario, Canada; Department of Cardiothoracic and Vascular Surgery, ${ }^{\mathrm{i}}$ Tromso University Hospital, Tromso, Norway; Department of Cardiovascular Surgery, ${ }^{\mathrm{j}}$ University of Pennsylvania School of Medicine, Philadelphia, Pa; Department of Cardiac Surgery, ${ }^{\mathrm{k}}$ University of Calgary Medical Center, Calgary, Alberta, Canada. management remains controversial because of the likely persistence (or progression) of neurologic deficit and considerable mortality and morbidity after surgery. ${ }^{3}$ Given the rare occurrence of brain complications at the onset of dissection, few (and conflicting) data exist, mostly derived from limited study populations. ${ }^{3-8}$ The International Registry for Acute Dissection (IRAD) was designed to enroll consecutive

Disclosures: Di Eusanio, Patel, Nienaber, Montgomery, Korach, Sundt, DeVincentiis, Voehringer, Peterson, Myrmel, Folesani, Larsen, Desai, Bavaria, Appoo, Kieser, Fattori, Eagle, Di Bartolomeo, and Trimarchi have nothing to disclose with regard to commercial support.

Presented at The American Association for Thoracic Surgery Aortic Surgery Symposium 2012, April 26-27, 2012, New York, NY.

Received for publication April 24, 2012; revisions received Aug 15, 2012; accepted for publication Nov 28, 2012.

Address for reprints: Marco Di Eusanio, MD, PhD, Department of Cardiac Surgery, Policlinico Sant'Orsola-Malpighi, Università di Bologna, Pad 25, Via Massarenti

9, Bologna 40128 Italy (E-mail: marco.dieusanio2@unibo.it).

$0022-5223 / \$ 36.00$

Copyright (c) 2013 by The American Association for Thoracic Surgery

http://dx.doi.org/10.1016/j.jtcvs.2012.11.054 


Abbreviations and Acronyms
$\begin{aligned} \mathrm{CI} & =\text { confidence interval } \\ \mathrm{CVA}= & \text { cerebrovascular accident } \\ \mathrm{IRAD}= & \text { International Registry for Acute } \\ & \text { Dissection } \\ \mathrm{OR}= & \text { odds ratio } \\ \mathrm{TA}-\mathrm{AAD}= & \text { type A acute aortic dissection }\end{aligned}$

patients from a broad geographic region to minimize the inherent biases seen in small surgical registries or singlecenter series. The aims of the present study were to compare the clinical and imaging characteristics, management, and short- and mid-term outcomes of a large group of patients with TA-AAD presenting with and without preoperative cerebrovascular accident (CVA) and coma and to assess the outcomes of patients with major brain injury according to different therapeutic strategies (surgical vs medical).

\section{METHODS \\ IRAD Registry and Data Collection}

The rationale and method of IRAD have been previously published. ${ }^{9}$ In brief, IRAD was founded by agreement of 18 large referral centers in 1996, with its main purpose being to assess the etiology, clinical features, imaging findings, treatment, and outcomes of patients with acute aortic dissection (both type A and type B). Patients with acute aortic dissection were identified either prospectively at presentation or retrospectively by searching hospital discharge diagnosis records and/or the surgery, pathology, and imaging databases. The diagnosis was determined from the imaging, surgical visualization, or autopsy findings. TA-AAD was defined as any dissection that involved the ascending aorta and/or aortic arch presenting within 14 days of symptom onset. Patient data were collected using standardized forms, including 290 variables for patient demographics, history, clinical presentation, physical findings, imaging studies, therapeutic management, in-hospital mortality, and adverse events. The completed data forms were forwarded by the participating IRAD sites to the coordinating center at the University of Michigan and reviewed for face validity and completeness. Annual follow-up data were obtained for up to 5 years after discharge using standardized data forms. The collected follow-up data included variables on clinical, imaging, and vital information. When applicable, missing data on mortality were obtained by searching the Social Security Death Index. At each enrolling hospital, the study investigators obtained approval from their ethics and/or institutional review board to participate in the IRAD studies.

\section{Patient Selection and Data Analysis}

We examined 1873 consecutive patients with TA-AAD enrolled from January 1996 to February 2011. The patients were categorized according to presence and type of preoperative major brain injury (no brain injury vs CVA vs coma). The preoperative clinical characteristics, imaging data, management, and mid-term outcomes were compared among the 3 subcohorts. CVA was defined as the persistent loss of neurologic function caused by an ischemic event, with or without confirmation by either computed tomography or magnetic resonance imaging. Coma was indicated if the patient experienced complete mental unresponsiveness (beyond that expected from anesthesia), with no evidence of psychological or physiologically appropriate responses to stimulation. A composite variable of adverse outcomes was created to report the incidence of the most severe and incapacitating complications after surgical repair: death and/or CVA and/or coma.

In patients with preoperative brain injury, the in-hospital outcomes were assessed according to type of management used (medical vs surgical), and the independent risk factors for hospital death were identified in all and in surgically treated patients with preoperative brain injury.

The estimates of 5-year survival were stratified by the presence and type of brain injury at admission (no brain injury vs CVA vs coma) in the overall population and in the subgroups of patients undergoing medical or surgical therapy. The independent predictors of follow-up mortality were identified in patients presenting with brain injury.

\section{Statistical Analysis}

Continuous variables are presented as the mean \pm standard deviation or median and quartile 1 to quartile 3 and categorical variables as percentages. In all cases, the missing data were not defaulted to negative, and the denominators reflected only the cases reported. Univariate analyses between groups were done using chi-square tests (or Fisher's exact tests) and Student's $t$ tests, as appropriate. Univariate analysis was first performed using preoperative and intraoperative variables to determine their individual relationship to in-hospital mortality in all patients and in surgically treated patients with preoperative brain injury. The variables that achieved $P<.15$ on univariate analysis and the variables from the previously published IRAD type A aortic dissection mortality mode ${ }^{10}$ were introduced to a gender-adjusted multivariate analysis by backward stepwise logistic regression to estimate the independent adjusted odds ratios (ORs) of factors related to hospital mortality. Long-term survival analysis was performed using Kaplan-Meier curves, and differences in survival between groups were examined with the log-rank test. Statistical analysis was performed using SPSS, version 18.0 (SPSS, Chicago, Ill).

\section{RESULTS}

\section{Characteristics of Patients With and Without Brain Injury}

Of 1873 patients with TA-AAD enrolled in IRAD, 87 $(4.7 \%)$ presented with CVA and $54(2.9 \%)$ with coma. Compared with the patients without brain injury, those with CVA and coma presented more rapidly to a referring or tertiary hospital (no brain injury, 2.1 hours; CVA, 1.1 hours; coma, 1.0 hour; $P<.001)$ and less frequently complained of chest pain (no brain injury, $81.7 \%$; CVA, $73.8 \%$; coma, 52.3\%; $P<.001$ ) or abdominal pain (no brain injury, $43.4 \%$; CVA, $32.5 \%$; coma, $34.1 \% ; P=.077)$ at the onset of dissection. Hypotension/shock/tamponade (no brain injury, $25.2 \%$; CVA, $40.2 \%$; coma, $59.3 \% ; P<.001$ ) and syncope (no brain injury, $15.3 \%$; CVA, $43.2 \%$; coma, $56.5 \%$; $P<.001$ ) were more likely to occur in patients with brain complications. Moreover, the risk profile of patients with CVA and coma was more often aggravated by renal failure (no brain injury, $7.9 \%$; CVA, $13.4 \%$; coma, $21.2 \% ; P=.002$ ), myocardial ischemia or infarction (no brain injury, $10.2 \%$; CVA, $13.4 \%$; coma, $25.0 \% ; P=.002$ ), and limb ischemia (no brain injury, 9.7\%; CVA, $18.3 \%$; coma, $15.4 \% ; P=.019$; Tables 1 and 2 ).

On the imaging studies, the characteristics of dissection were similar in the patients with and without brain injury, except for arch vessel involvement by the dissection, which was documented in $61.8 \%$ of patients with CVA, $43.6 \%$ of patients with coma, and $36.1 \%$ of uncomplicated patients $(P<.001$; Table 3$)$. 
TABLE 1. Demographics and history of patients with and without major brain injury (CVA and coma)

\begin{tabular}{|c|c|c|c|c|}
\hline Variable & $\begin{array}{c}\text { No brain } \\
\text { injury }\end{array}$ & CVA & Coma & $\begin{array}{c}P \\
\text { value }\end{array}$ \\
\hline Age (y) & $61.5 \pm 14.6$ & $63.1 \pm 13.7$ & $62.8 \pm 12.2$ & .506 \\
\hline Men & $1177(67.9 \%)$ & $54(62.1 \%)$ & $31(57.4 \%)$ & .151 \\
\hline White race & $1449(89.7 \%)$ & $78(95.1 \%)$ & $47(90.4 \%)$ & .281 \\
\hline $\begin{array}{l}\text { Interval from } \\
\text { symptoms to } \\
\text { presentation (h) }\end{array}$ & $2.1(1.0-11.9)$ & $1.1(0.7-2.9)$ & $1.0(0.5-1.9)$ & $<.001$ \\
\hline $\begin{array}{l}\text { Interval from } \\
\text { presentation } \\
\text { to surgery (h) }\end{array}$ & $12.8(5.7-30.7)$ & $9.8(4.4-54.6)$ & $9.7(5.1-20.0)$ & .494 \\
\hline $\begin{array}{l}\text { Interval from } \\
\text { symptoms } \\
\text { to surgery (h) }\end{array}$ & $15.9(7.5-42.7)$ & $12.3(6.6-56.1)$ & $13.8(6.3-24.0)$ & .310 \\
\hline \multicolumn{5}{|l|}{ Etiology and history } \\
\hline Atherosclerosis & $369(22.1 \%)$ & $28(32.6 \%)$ & $13(25.5 \%)$ & .072 \\
\hline Diabetes & $103(6.2 \%)$ & $4(4.7 \%)$ & $4(7.8 \%)$ & .737 \\
\hline Hypertension & $1192(70.6 \%)$ & $68(80.0 \%)$ & $33(66.0 \%)$ & .131 \\
\hline $\begin{array}{c}\text { Aortic valve } \\
\text { disease }\end{array}$ & $205(12.3 \%)$ & $10(11.9 \%)$ & $5(10.0 \%)$ & .882 \\
\hline $\begin{array}{l}\text { Bicuspid aortic } \\
\text { valve }\end{array}$ & $65(4.5 \%)$ & $4(5.6 \%)$ & $2(4.8 \%)$ & .701 \\
\hline Marfan & $78(4.7 \%)$ & $3(3.5 \%)$ & $0(0.0 \%)$ & .358 \\
\hline Peripartum & $4(0.2 \%)$ & $1(1.2 \%)$ & $0(0.0 \%)$ & .329 \\
\hline Cocaine abuse & $23(1.4 \%)$ & $1(1.2 \%)$ & $0(0.0 \%)$ & 1.000 \\
\hline $\begin{array}{c}\text { Known aortic } \\
\text { aneurysm }\end{array}$ & $219(13.1 \%)$ & $9(10.6 \%)$ & $4(8.0 \%)$ & .466 \\
\hline $\begin{array}{l}\text { Previous aortic } \\
\text { dissection }\end{array}$ & $69(4.1 \%)$ & $2(2.4 \%)$ & $0(0.0 \%)$ & .355 \\
\hline $\begin{array}{l}\text { Previous cardiac } \\
\text { surgery }\end{array}$ & $236(14.3 \%)$ & $12(14.5 \%)$ & $6(11.8 \%)$ & .879 \\
\hline $\begin{array}{l}\text { History of } \\
\text { catheterization/ } \\
\text { angiography }\end{array}$ & $162(11.5 \%)$ & $6(9.1 \%)$ & $3(7.3 \%)$ & .752 \\
\hline
\end{tabular}

\section{Therapeutic Strategies for Patients With and Without Brain Injury}

Surgical management was avoided more often in patients with coma $(33.3 \%)$ or stroke $(24.1 \%)$ than in those without brain injury $(11.1 \% ; P<.001)$. The reasons given for medical management included 1 or more of the following: comorbid illness, advanced age, and patient or family refusal. In surgically treated patients, the extent of aortic replacement, rate of associated cardiac procedures, and rate of open aortic anastomosis were equally distributed in patients with and without a brain deficit (Table 4).

\section{Hospital Outcomes: Preoperative Brain Injury Versus No Brain Injury}

Overall, hospital mortality was $22.7 \%$ in patients without a preoperative brain deficit, $40.2 \%$ in patients with CVA,
TABLE 2. Clinical presentation of patients with and without major brain injury (CVA and coma)

\begin{tabular}{|c|c|c|c|c|}
\hline Variable & $\begin{array}{l}\text { No brain } \\
\text { injury }\end{array}$ & CVA & Coma & $\begin{array}{c}P \\
\text { value }\end{array}$ \\
\hline Chest pain & $1368(81.7 \%)$ & $62(73.8 \%)$ & $23(52.3 \%)$ & $<.001$ \\
\hline Anterior & $1105(80.0 \%)$ & $55(78.6 \%)$ & $19(52.8 \%)$ & $<.001$ \\
\hline Posterior & $499(39.4 \%)$ & $26(40.0 \%)$ & $8(24.2 \%)$ & .209 \\
\hline Back pain & $702(43.4 \%)$ & $27(32.5 \%)$ & $15(34.1 \%)$ & .077 \\
\hline Abdominal pain & $426(26.5 \%)$ & $15(18.5 \%)$ & $12(26.7 \%)$ & .282 \\
\hline Leg pain & $189(11.9 \%)$ & $13(16.3 \%)$ & $6(14.0 \%)$ & .474 \\
\hline \multicolumn{5}{|l|}{ Quality of pain } \\
\hline Migrating & $194(12.4 \%)$ & $16(20.3 \%)$ & $4(9.5 \%)$ & .102 \\
\hline Radiating & $602(38.1 \%)$ & $16(20.3 \%)$ & $12(28.6 \%)$ & .003 \\
\hline \multicolumn{5}{|l|}{ Pain severity } \\
\hline Mild & $110(8.0 \%)$ & $6(9.0 \%)$ & $2(6.7 \%)$ & .950 \\
\hline Severe & $1044(76.3 \%)$ & $52(77.6 \%)$ & $19(63.3 \%)$ & .246 \\
\hline Worst ever & $214(15.6 \%)$ & $9(13.4 \%)$ & $9(30.0 \%)$ & .104 \\
\hline Abrupt onset of pain & $1340(82.7 \%)$ & $67(83.8 \%)$ & $34(81.0 \%)$ & .927 \\
\hline Febrile & $33(2.4 \%)$ & $2(3.2 \%)$ & $2(5.9 \%)$ & .219 \\
\hline $\begin{array}{l}\text { Hypotension/ } \\
\text { shock/tamponade }\end{array}$ & $403(25.2 \%)$ & $33(40.2 \%)$ & $32(59.3 \%)$ & $<.001$ \\
\hline Hypertension & $501(31.4 \%)$ & $21(25.6 \%)$ & $10(18.5 \%)$ & .079 \\
\hline Syncope & $252(15.3 \%)$ & $35(43.2 \%)$ & $26(56.5 \%)$ & $<.001$ \\
\hline $\begin{array}{l}\text { Ischemic spinal cord } \\
\text { damage }\end{array}$ & $25(1.6 \%)$ & $2(2.4 \%)$ & $2(4.3 \%)$ & .131 \\
\hline $\begin{array}{l}\text { Myocardial ischemia/ } \\
\text { infarction }\end{array}$ & $172(10.2 \%)$ & $11(13.4 \%)$ & $13(25.0 \%)$ & .002 \\
\hline Cardiac heart failure & $112(6.7 \%)$ & $7(8.3 \%)$ & $7(14.0 \%)$ & .127 \\
\hline Acute renal failure & $134(7.9 \%)$ & $11(13.4 \%)$ & $11(21.2 \%)$ & .002 \\
\hline Limb ischemia & $163(9.7 \%)$ & $15(18.3 \%)$ & $8(15.4 \%)$ & .019 \\
\hline Any pulse deficit & $363(28.1 \%)$ & $40(52.6 \%)$ & $18(43.9 \%)$ & $<.001$ \\
\hline
\end{tabular}

and $63.0 \%$ in those with coma $(P<.001)$. After treatment (either medical or surgical), CVA and coma were noted in $8.1 \%$ and $3.0 \%$ of patients presenting without brain injury, $26.7 \%$ and $5.0 \%$ of patients presenting with CVA, and $9.4 \%$ and $21.9 \%$ of patients presenting with coma, respectively $(P<.001)$. Myocardial infarction or ischemia (no brain injury, $13.9 \%$; CVA, $17.7 \%$; coma, $32.1 \%$; $P=.001$ ) and limb ischemia (no brain injury, $10.1 \%$; CVA, $19.0 \%$; coma, $19.2 \% ; P=.006$ ) were more likely to occur in patients with preoperative brain injury than in those without (Table 5).

\section{Hospital Outcomes in Patients With Preoperative Brain Injury: Medical Versus Surgical Management}

All patients (100\%) presenting with coma and $76.2 \%$ of those with CVA died when medical management was undertaken. Only $5(12.8 \%)$ of the 39 medically managed patients with preoperative brain injury survived to discharge. Alternatively, after surgery, mortality was $27.0 \%$ for the patients with CVA and $44.0 \%$ for those with preoperative coma $(P<.001)$. Of these 99 patients with 
TABLE 3. Characteristics of dissection in patients with and without mesenteric ischemia

\begin{tabular}{|c|c|c|c|c|}
\hline Variable & $\begin{array}{c}\text { No brain } \\
\text { injury }\end{array}$ & CVA & Coma & $\begin{array}{c}P \\
\text { value }\end{array}$ \\
\hline \multicolumn{5}{|l|}{ Origin of dissection flap } \\
\hline $\begin{array}{l}\text { Sinotubular } \\
\text { junction }\end{array}$ & $197(12.1 \%)$ & $7(8.2 \%)$ & $5(10.0 \%)$ & .512 \\
\hline Aortic root & $786(48.4 \%)$ & $42(49.4 \%)$ & $25(50.0 \%)$ & .959 \\
\hline Ascending & $540(33.2 \%)$ & $31(36.5 \%)$ & $17(34.0 \%)$ & .823 \\
\hline Arch & $64(3.9 \%)$ & $5(5.9 \%)$ & $0(0.0 \%)$ & .210 \\
\hline \multicolumn{5}{|l|}{ Site of intimal tear } \\
\hline Multiple & $44(3.6 \%)$ & $1(1.4 \%)$ & $2(5.4 \%)$ & .507 \\
\hline Not identified & $481(39.8 \%)$ & $32(46.4 \%)$ & $16(43.2 \%)$ & .546 \\
\hline Descending & $25(2.1 \%)$ & $0(0.0 \%)$ & $0(0.0 \%)$ & .695 \\
\hline Ascending & $569(47.0 \%)$ & $30(43.5 \%)$ & $19(51.4 \%)$ & .732 \\
\hline Arch & $91(7.5 \%)$ & $6(8.7 \%)$ & $0(0.0 \%)$ & .187 \\
\hline \multicolumn{5}{|l|}{ False lumen patency } \\
\hline Patent & $768(71.2 \%)$ & $46(68.7 \%)$ & $26(81.3 \%)$ & .411 \\
\hline Partial thrombosis & $214(19.9 \%)$ & $15(22.4 \%)$ & $4(12.5 \%)$ & .507 \\
\hline $\begin{array}{l}\text { Complete } \\
\text { thrombosis }\end{array}$ & $96(8.9 \%)$ & $6(9.0 \%)$ & $2(6.3 \%)$ & 1.000 \\
\hline $\begin{array}{l}\text { Arch vessels } \\
\text { involvement }\end{array}$ & $449(36.1 \%)$ & $42(61.8 \%)$ & $17(43.6 \%)$ & $<.001$ \\
\hline $\begin{array}{l}\text { Any abdominal artery } \\
\text { involvement }\end{array}$ & $341(19.9 \%)$ & $29(33.7 \%)$ & $18(34.0 \%)$ & .001 \\
\hline $\begin{array}{c}\text { Coronary arteries } \\
\text { compromised }\end{array}$ & $161(12.8 \%)$ & $7(10.1 \%)$ & $7(17.5 \%)$ & .542 \\
\hline Aortic regurgitation & $794(55.3 \%)$ & $39(53.4 \%)$ & $15(31.9 \%)$ & .006 \\
\hline \multicolumn{5}{|c|}{ Aortic measurement $(\mathrm{cm})$} \\
\hline Aortic annulus & $2.5(2.3-2.9)$ & $2.6(2.3-2.9)$ & $2.4(2.2-2.6)$ & .215 \\
\hline Aortic root & $4.2(3.7-5.0)$ & $4.2(3.6-4.7)$ & $4.2(3.8-5.0)$ & .905 \\
\hline $\begin{array}{l}\text { Ascending aorta } \\
\text { (widest) }\end{array}$ & $5.0(4.5-5.8)$ & $4.8(4.0-5.5)$ & $4.9(4.5-5.4)$ & .379 \\
\hline Aortic arch & $3.7(3.2-4.1)$ & $3.5(3.1-4.3)$ & $3.8(3.5-4.0)$ & .481 \\
\hline $\begin{array}{l}\text { Descending aorta } \\
\text { (widest) }\end{array}$ & $3.4(3.0-3.9)$ & $3.4(3.0-3.9)$ & $3.1(2.8-3.5)$ & .950 \\
\hline
\end{tabular}

Data presented as $\mathrm{n}(\%)$ or median (quartile 1 to quartile 3). CVA, Cerebrovascular accident.

preoperative brain injury, $66(66.7 \%)$ survived to discharge. Postoperatively, brain injury reversal occurred in $80.4 \%$ and $74.2 \%$ of those with CVA and coma, respectively. Thus, the composite adverse outcome occurred in $36.5 \%$ and $50.0 \%$ of those with CVA and coma, respectively (Table 6).

\section{Independent Predictors of Hospital Mortality in Patients With Preoperative Brain Injury}

On binary logistic regression analysis, surgery was protective against mortality in patients with preoperative brain injury (CVA or coma; OR, $0.058 ; 95 \%$ confidence interval [CI], 0.018-0.192; $P<.001)$. In contrast, hypotension/ shock/tamponade (OR, 3.4; 95\% CI, 1.365-8.415; $P=.009)$ and renal failure (OR, 3.4; 95\% CI, 1.292 9.159; $P=.013)$ were independent risk factors for reduced hospital survival (Figure 1).
TABLE 4. Therapeutic management and surgical procedures for patients with type $\mathrm{A}$ acute dissection with and without major brain injury

\begin{tabular}{|c|c|c|c|c|}
\hline Variable & $\begin{array}{c}\text { No brain } \\
\text { injury }\end{array}$ & CVA & Coma & $\begin{array}{c}P \\
\text { value }\end{array}$ \\
\hline \multicolumn{5}{|c|}{ Therapeutic management } \\
\hline Surgical & $1496(86.3 \%)$ & $63(72.4 \%)$ & $36(66.7 \%)$ & $<.001$ \\
\hline Medical & $192(11.1 \%)$ & $21(24.1 \%)$ & $18(33.3 \%)$ & $<.001$ \\
\hline \multicolumn{5}{|c|}{ Extent of aortic replacement } \\
\hline $\begin{array}{l}\text { Ascending aorta } \\
\text { replacement }\end{array}$ & $1361(93.2 \%)$ & $59(92.2 \%)$ & $33(94.3 \%)$ & .897 \\
\hline Root replacement & $488(36.7 \%)$ & $17(30.9 \%)$ & $8(25.8 \%)$ & .352 \\
\hline $\begin{array}{r}\text { Complete arch } \\
\text { replacement }\end{array}$ & $229(16.0 \%)$ & $8(13.3 \%)$ & $4(11.8 \%)$ & .783 \\
\hline $\begin{array}{l}\text { Partial arch } \\
\text { replacement }\end{array}$ & $560(39.5 \%)$ & $30(50.0 \%)$ & $12(36.4 \%)$ & .247 \\
\hline Open procedure & $773(90.0 \%)$ & $36(90.0 \%)$ & $19(86.4 \%)$ & .739 \\
\hline Reoperation & $180(12.9 \%)$ & $7(11.9 \%)$ & $2(6.1 \%)$ & .625 \\
\hline \multicolumn{5}{|l|}{ Associated procedures } \\
\hline CABG & $166(11.7 \%)$ & $3(4.8 \%)$ & $6(17.1 \%)$ & .128 \\
\hline MVR & $8(0.6 \%)$ & $0(0.0 \%)$ & $0(0.0 \%)$ & 1.000 \\
\hline AVR & $397(27.8 \%)$ & $12(19.7 \%)$ & $9(25.7 \%)$ & .367 \\
\hline $\begin{array}{l}\text { Peripheral vessels } \\
\text { replaced }\end{array}$ & $71(5.0 \%)$ & $4(6.6 \%)$ & $0(0.0 \%)$ & .360 \\
\hline
\end{tabular}

Data presented as n (\%). CVA, Cerebrovascular accident; $C A B G$, coronary artery bypass grafting; $M V R$, mitral valve replacement; $A V R$, aortic valve replacement.

\section{Independent Predictors of Hospital Mortality in Surgical Patients With Preoperative Brain Injury}

In surgically treated patients, the only independent predictor of hospital death was hypotension/shock/tamponade (OR, 5.4; 95\% CI, 0.018-0.192; $P=.009$ ); however, brain injury reversal was protective against hospital mortality (OR, 0.014; 95\% CI, 0.069-0.742; $P=.226$; Figure 2). The delay from the onset of dissection and surgery did not correlate with mortality and brain injury reversal.

\section{Follow-up Mortality}

Of 1411 patients, $705(50.0 \%)$ had follow-up death data available. The median follow-up duration was 36 months (quartile 1 to quartile 3, 1.02-60.0 months). The KaplanMeier estimate of 1-, 3-, and 5-year survival was $76.5 \%$, $74.1 \%$, and $72.9 \%$ for patients presenting without brain injury, $57.0 \%, 55.4 \%$, and $55.4 \%$ for those with preoperative CVA, and $37.7 \%, 37.7 \%$, and $37.7 \%$ for those with preoperative coma, respectively (log-rank, $P<.001$; Figure 3 , $A$ ). The mid-term estimates of postadmission survival for patients receiving medical and surgical management are depicted in Figure 3, B,C: the 5-year survival of patients presenting with CVA and coma was $23.8 \%$ and $0 \%$ after medical management and $67.1 \%$ and $57.1 \%$ after surgery.

\section{Independent Predictors of Follow-up Mortality in Patients With Preoperative Brain Injury}

In patients with TA-AAD complicated by preoperative brain injury, Cox proportional hazards regression analysis 
TABLE 5. Overall in-hospital mortality and complications for patients with type $A$ acute dissection patients with and without major brain injury

\begin{tabular}{|c|c|c|c|c|}
\hline Result & $\begin{array}{l}\text { No brain } \\
\text { injury }\end{array}$ & CVA & Coma & $\begin{array}{c}P \\
\text { value }\end{array}$ \\
\hline Discharged home & $1141(74.8 \%)$ & $38(54.3 \%)$ & $17(39.5 \%)$ & $<.001$ \\
\hline Mortality & $394(22.7 \%)$ & $35(40.2 \%)$ & $34(63.0 \%)$ & $<.001$ \\
\hline CVA & $177(8.1 \%)$ & $16(26.7 \%)$ & $3(9.4 \%)$ & $<.001$ \\
\hline Coma & $44(3.0 \%)$ & $3(5.0 \%)$ & $7(21.9 \%)$ & $<.001$ \\
\hline Spinal cord injury & $55(3.4 \%)$ & $4(5.4 \%)$ & $2(4.1 \%)$ & .479 \\
\hline $\begin{array}{l}\text { Myocardial infarction/ } \\
\text { ischemia }\end{array}$ & $231(13.9 \%)$ & $14(17.7 \%)$ & $17(32.1 \%)$ & .001 \\
\hline Acute renal failure & $362(21.9 \%)$ & $19(24.1 \%)$ & $17(32.7 \%)$ & .168 \\
\hline Limb ischemia & $166(10.1 \%)$ & $15(19.0 \%)$ & $10(19.2 \%)$ & .006 \\
\hline Cardiac tamponade & $289(17.5 \%)$ & $16(20.3 \%)$ & $14(26.9 \%)$ & .189 \\
\hline
\end{tabular}

Data presented as n (\%). CVA, Cerebrovascular accident.

indicated hypotension/shock/tamponade (hazard ratio, 6.2; 95\% CI, 2.710-14.348; $P<.001$ ) and renal failure (hazard ratio, $1.9 ; 95 \% \mathrm{CI}, 1.029-3.768 ; P=.041)$ as significant independent predictors of reduced 5-year postadmission survival. In contrast, surgical management (hazard ratio, 0.084; 95\% CI, $0.035-0.202 ; P<.001)$ was protective against follow-up mortality (Table E1).

\section{DISCUSSION}

\section{Risk Profile and Outcomes of Patients With TA-AAD}

\section{Presenting With Brain Injury}

Different pathogenetic mechanisms could determine CVA and coma in patients with TA-AAD. These include brain malperfusion from occlusion of the arch vessels by

TABLE 6. Hospital outcomes of patients with brain injury stratified by treatment strategy

\begin{tabular}{|c|c|c|c|c|c|c|}
\hline \multirow[b]{2}{*}{ Outcome } & \multicolumn{3}{|c|}{ CVA } & \multicolumn{3}{|c|}{ Coma } \\
\hline & Medical & Surgical & $\begin{array}{c}P \\
\text { value } \\
\end{array}$ & Medical & Surgical & $\begin{array}{c}P \\
\text { value } \\
\end{array}$ \\
\hline Mortality & $16(76.2)$ & $17(27.0)$ & $<.001$ & $18(100.0)$ & $16(44.4)$ & $<.001$ \\
\hline $\begin{array}{l}\text { Discharged } \\
\text { home }\end{array}$ & $3(21.4)$ & $34(61.8)$ & .007 & $0(0.0)$ & 17 (58.6) & $<.001$ \\
\hline CVA & NA & $8(15.7)$ & & NA & $3 / 31(9.7)$ & \\
\hline Coma & NA & $2(3.9)$ & & NA & $7 / 31(22.6)$ & \\
\hline $\begin{array}{l}\text { Brain injury } \\
\text { reversal }\end{array}$ & NA & $4(80.4)$ & & NA & $23 / 31(74.2)$ & \\
\hline $\begin{array}{l}\text { Spinal cord } \\
\text { injury }\end{array}$ & $1(4.8)$ & $3(5.7)$ & 1.000 & $2(11.1)$ & $0(0.0)$ & .130 \\
\hline $\begin{array}{l}\text { Myocardial } \\
\text { infarction/ } \\
\text { ischemia }\end{array}$ & $5(26.3)$ & $9(15.0)$ & .306 & $4(23.5)$ & $13(36.1)$ & .530 \\
\hline $\begin{array}{l}\text { Acute renal } \\
\text { failure }\end{array}$ & $6(31.6)$ & $13(21.7)$ & .374 & $5(29.4)$ & $12(34.3)$ & .725 \\
\hline $\begin{array}{l}\text { Limb } \\
\text { ischemia }\end{array}$ & $2(10.5)$ & $13(21.7)$ & .502 & $5(29.4)$ & $5(14.3)$ & .264 \\
\hline $\begin{array}{l}\text { Cardiac } \\
\text { tamponade }\end{array}$ & $2(10.5)$ & $14(23.3)$ & .332 & $4(23.5)$ & $10(28.6)$ & 1.000 \\
\hline
\end{tabular}

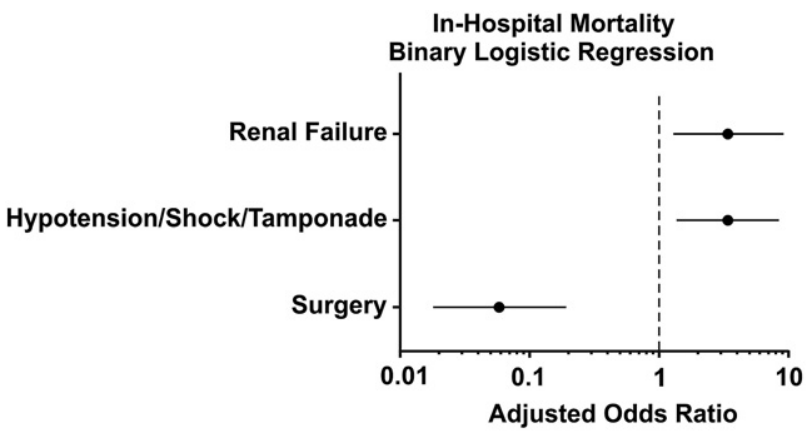

FIGURE 1. On binary logistic regression analysis, surgery was protective against mortality in patients with preoperative brain injury (cerebrovascular accident or coma; odds ratio, $0.058 ; 95 \%$ confidence interval, 0.018 0.192; $P<.001$ ), but hypotension/shock/tamponade (odds ratio, 3.4; 95\% confidence interval, 1.365-8.415; $P=.009$ ) and renal failure (odds ratio, 3.4; 95\% confidence interval, $1.292-9.159 ; P=.013$ ) were independent risk factors for reduced hospital survival.

the intimal-medial flap, hypoxic encephalopathy secondary to shock/tamponade, brain embolism from thrombus in the false lumen, and/or a combination of several of these. The IRAD data from 1873 patients with TA-AAD indicated preoperative major brain injury occurred in approximately $8 \%$ of patients (CVA, 4.7\%; coma, 2.9\%), and hypotension/ shock/tamponade and arch vessel involvement were strongly associated with CVA and coma at presentation. Although thromboembolic and composite etiologies of brain injury could not be assessed in our study, univariate analysis showed a significantly greater incidence of hypotension/ shock/tamponade $(59.3 \%$ vs $25.2 \%)$ and arch vessel involvement $(61 \%$ vs $36.1 \%)$ in patients with coma and CVA, respectively, than in patients without any brain injury. Furthermore, renal failure, myocardial infarction/ischemia, limb ischemia, any pulse deficit, and any abdominal artery involvement were significantly more prevalent in patients with CVA and coma, confirming the predominant and detrimental role that hypotension/shock/tamponade and malperfusion have in the clinical presentation (and prognosis) of

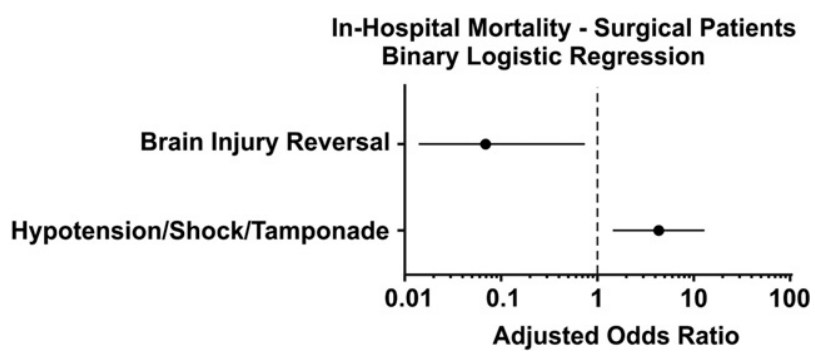

FIGURE 2. In surgically treated patients, the only independent predictor of hospital death was hypotension/shock/tamponade (odds ratio, 5.4; 95\% confidence interval, 0.018-0.192; $P=.009$ ); however, brain injury reversal was protective against hospital mortality (odds ratio, $0.014 ; 95 \%$ confidence interval, 0.069-0.742; $P=.226$ ). 

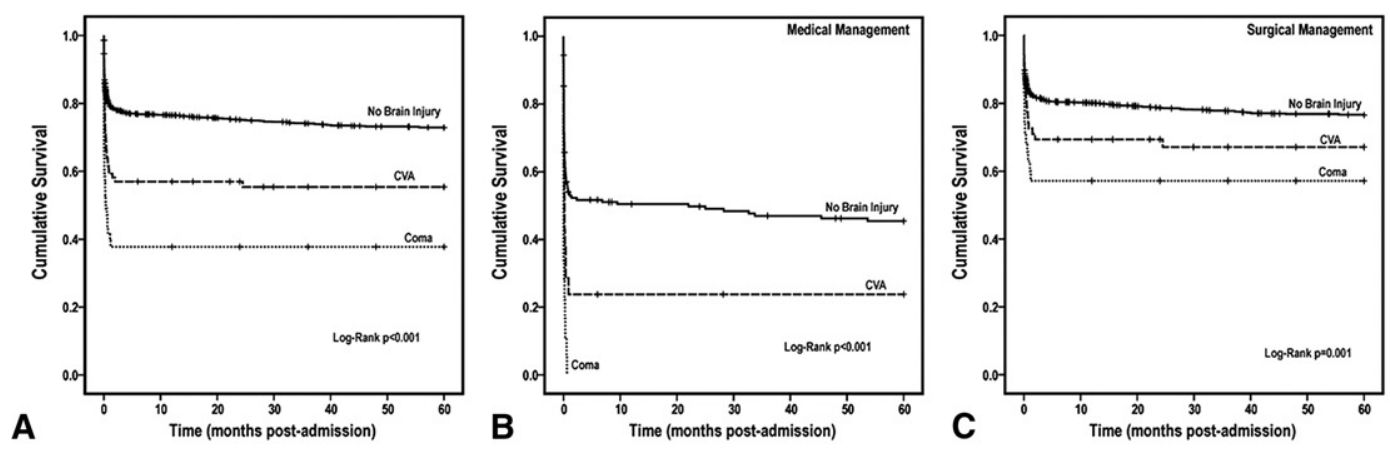

A. All Patients: 5-Year Post-Admission Survival.

\begin{tabular}{|l|c|c|c|c|c|}
\hline $\begin{array}{l}\text { Survival } \\
\text { Estimate }\end{array}$ & 1-Year & 2-Year & 3-Year & 4-Year & 5-Year \\
\hline No Brain Injury & $76.5 \pm 1.0 \%$ & $75.2 \pm 1.1 \%$ & $74.1 \pm 1.1 \%$ & $73.2 \pm 1.1 \%$ & $72.9 \pm 1.1 \%$ \\
\hline Total Events & 393 & 411 & 420 & 435 & 439 \\
\hline At Risk & 1200 & 1017 & 937 & 838 & 770 \\
\hline Preop CVA & $57.0 \pm 5.3 \%$ & $57.0 \pm 5.3 \%$ & $55.4 \pm 5.4 \%$ & $55.4 \pm 5.4 \%$ & $55.4 \pm 5.4 \%$ \\
\hline Total Events & 37 & 37 & 38 & 38 & 38 \\
\hline At Risk & 46 & 39 & 32 & 28 & 26 \\
\hline Preop Coma & $37.7 \pm 6.7 \%$ & $37.7 \pm 6.7 \%$ & $37.7 \pm 6.7 \%$ & $37.7 \pm 6.7 \%$ & $37.7 \pm 6.7 \%$ \\
\hline Total Events & 33 & 33 & 33 & 33 & 33 \\
\hline At Risk & 20 & 17 & 16 & 13 & 11 \\
\hline
\end{tabular}

B. Medical Management Patients Only: 5-Year Post-Admission Survival.

\begin{tabular}{|l|c|c|c|c|c|}
\hline $\begin{array}{l}\text { Survival } \\
\text { Estimate }\end{array}$ & 1-Year & 2-Year & 3-Year & 4-Year & 5-Year \\
\hline No Brain Injury & $50.4 \pm 3.8 \%$ & $49.7 \pm 3.8 \%$ & $47.0 \pm 3.8 \%$ & $46.2 \pm 3.9 \%$ & $45.4 \pm 3.9 \%$ \\
\hline Total Events & 86 & 87 & 91 & 92 & 93 \\
\hline At Risk & 80 & 72 & 67 & 60 & 55 \\
\hline Preop CVA & $23.8 \pm 9.3 \%$ & $23.8 \pm 9.3 \%$ & $23.8 \pm 9.3 \%$ & $23.8 \pm 9.3 \%$ & $23.8 \pm 9.3 \%$ \\
\hline Total Events & 16 & 16 & 16 & 16 & 16 \\
\hline At Risk & 4 & 4 & 3 & 3 & 2 \\
\hline Preop Coma & $0.0 \%$ & $0.0 \%$ & $0.0 \%$ & $0.0 \%$ & $0.0 \%$ \\
\hline Total Events & 18 & 18 & 18 & 18 & 18 \\
\hline At Risk & 0 & 0 & 0 & 0 & 0 \\
\hline
\end{tabular}

C. Surgical Management Patients Only: 5-Year Post-Admission Survival.

\begin{tabular}{|l|c|c|c|c|c|}
\hline $\begin{array}{l}\text { Survival } \\
\text { Estimate }\end{array}$ & 1-Year & 2-Year & 3-Year & 4-Year & 5-Year \\
\hline No Brain Injury & $80.1 \pm 1.0 \%$ & $78.7 \pm 1.1 \%$ & $77.8 \pm 1.1 \%$ & $76.9 \pm 1.1 \%$ & $76.5 \pm 1.1 \%$ \\
\hline Total Events & 289 & 306 & 316 & 325 & 328 \\
\hline At Risk & 1091 & 919 & 836 & 754 & 691 \\
\hline Preop CVA & $69.4 \pm 5.9 \%$ & $69.4 \pm 5.9 \%$ & $67.1 \pm 6.1 \%$ & $67.1 \pm 6.1 \%$ & $67.1 \pm 6.1 \%$ \\
\hline Total Events & 19 & 19 & 20 & 20 & 20 \\
\hline At Risk & 41 & 34 & 28 & 24 & 22 \\
\hline Preop Coma & $57.1 \pm 8.4 \%$ & $57.1 \pm 8.4 \%$ & $57.1 \pm 8.4 \%$ & $57.1 \pm 8.4 \%$ & $57.1 \pm 8.4 \%$ \\
\hline Total Events & 15 & 15 & 15 & 15 & 15 \\
\hline At Risk & 19 & 17 & 14 & 13 & 11 \\
\hline
\end{tabular}

FIGURE 3. Kaplan-Meier survival curves stratified by presence and type of brain injury in A, overall population of patients with type A acute dissection, $\mathrm{B}$, patients receiving medical management, and $\mathrm{C}$, patients undergoing surgical repair. CVA, Cerebrovascular accident. 
patients with brain injury. ${ }^{11}$ Hence, hospital mortality was twofold $(40.2 \%)$ and threefold $(63.0 \%)$ greater in patients presenting with CVA and coma, respectively, than in those without any brain injury $(22.7 \%)$. Hypotension/shock/tamponade and renal failure, with a similar OR of 3.4, were strong predictors of reduced hospital survival in patients presenting with major brain injury. ${ }^{10}$

\section{Therapeutic Management and Outcomes of Patients With TA-AAD Presenting With Brain Injury}

TA-AAD is a lethal condition, and, although no randomized studies of medical versus surgical management have been performed, it represents an accepted indication for emergent surgery, which can convert a $90 \%$ mortality risk to at least a $70 \%$ chance of survival. ${ }^{12}$ However, because cerebral reperfusion and hemorrhagic conversion of the ischemic region might worsen neurologic outcomes and lead to prohibitive postoperative mortality and morbidity rates, the pivotal role of surgery is questioned when serious preoperative brain injuries are present and remains controversial. ${ }^{3-5,12,13}$ In the IRAD centers, the presence and type of brain injury significantly affected the therapeutic management. Surgery was not performed in $11 \%$ of patients without brain injury, $24.1 \%$ of patients with CVA, and $33.3 \%$ of patients with coma, echoing a surgical preference to sidestep surgery for these highrisk patients.

When assessing hospital outcomes according to therapeutic management, however, our data showed that medical therapy was associated with dismal outcomes: $100 \%$ mortality in patients with coma and $76.2 \%$ in those with CVA. Thus, surgery led to a hospital survival benefit of $49.6 \%$ in patients with preoperative CVA and $55.6 \%$ of those with coma. Only $12.8 \%$ of the medically treated patients with preoperative brain injury survived to discharge compared with $66.7 \%$ of those undergoing surgical repair. Hospital mortality after surgical treatment was particularly satisfactory in those with CVA $(27.0 \%)$, not deviating markedly from that observed in patients without brain injury in the present series $(22.7 \%)$ or from those previously reported by IRAD and other registries of aortic dissection. ${ }^{14,15}$ Moreover, surgical survival benefits were maintained at 5 years, with curves parallel during follow-up (Figure 4). Such observations were also supported by multivariate and Cox analyses, suggesting surgery as a protective factor against both hospital and follow-up mortality in patients with preoperative brain injury.

We are well aware that our study had all the limitations inherent to registry data analysis and that patients deemed operable at the IRAD sites likely presented with a more favorable risk profile than those who were treated medically and, therefore, associated with the best hospital and midterm outcomes. Nevertheless, the hospital and 5-year survival benefits of surgery compared with medical therapy were dramatic and, therefore, important to consider.

\section{Brain Injury Reversal}

Urgent repair in patients with TA-AAD presenting with major brain injury might be tempered by the concern that immediate cerebral reperfusion could worsen neurologic outcomes. Cambria and colleagues ${ }^{3}$ reported that 6 of 7 patients with preoperative brain infarction died of brain damage after aortic dissection repair. However, in a series of 14 patients with preoperative stroke and aortic dissection, Estrera and colleagues ${ }^{4}$ recently reported hospital mortality of $7.0 \%$, with neurologic status completely recovered in $14 \%$, improved in $43 \%$, unvaried in $43 \%$, and worsened in $0 \%$ after surgery. Early surgical repair (within 10 hours) correlated with improvement in neurologic status. Similarly, Tsukube and colleagues, ${ }^{6}$ in 27 patients with TA-AAD presenting with coma, reported hospital mortality of $14 \%$ after early surgery, with full recovery of consciousness achieved in $86 \%$ of patients and complete independence in daily activities reached in $52 \%$ of survivors at 3 years. Our data from 141 patients with TA-AAD presenting with CVA or coma strongly support those reported by Estrera and colleagues ${ }^{4}$ and Tsukube and colleagues, ${ }^{6}$ showing that brain injury reversal is likely after surgery in selected patients, occurring in $80.4 \%$ and $74.2 \%$ of our patients with CVA and coma, respectively.

\section{Study Limitations and Strengths}

Our definitions of stroke and coma were based on a general clinical assessment and not specific quantitative or qualitative clinical criteria, such as the Glasgow Coma Scale, National Institutes of Health Stroke Scale, Rankin Score, or radiologic tests. We were not able to distinguish between patients with different cerebral lesions by location, size, or clinical relevance. Thus, the comparison of the short- and mid-term outcomes between surgical and medical management might have been influenced by a selection bias in the therapeutic referral process.

Only recently, IRAD has developed an "invasive treatment data form," aiming to collect detailed information about the surgical techniques and strategies used by IRAD surgeons. Thus, data on cannulation techniques, methods of brain protection, and techniques for arch (and arch vessel) repair were sparse and not sufficient to indicate their potential effect on surgical outcomes.

Only $50 \%$ of our patients had follow-up data available, an additional limitation of our study. Nevertheless, our estimate of follow-up survival was in line with that previously reported by our registry. ${ }^{16}$ Our follow-up did not address the quality of life of the discharged patients with preoperative brain injury.

The current knowledge of patients with TA-AAD complicated by brain injury is limited and mostly from small surgical case studies. Our study assessed the largest series of 


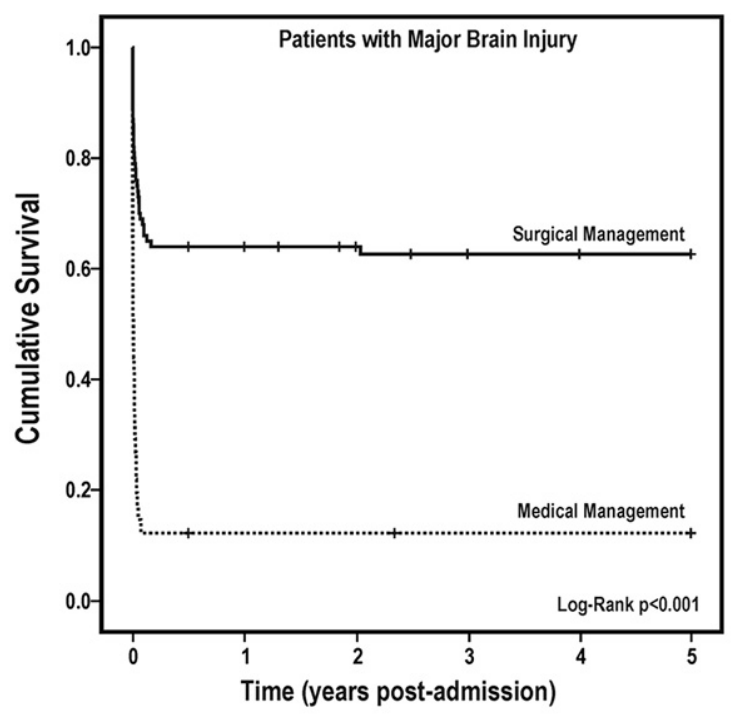

\begin{tabular}{|c|c|c|c|c|c|}
\hline $\begin{array}{l}\text { Survival } \\
\text { Estimate }\end{array}$ & 1-Year & 2-Year & 3-Year & 4-Year & 5-Year \\
\hline $\begin{array}{c}\text { Medical } \\
\text { Management }\end{array}$ & $12.2 \pm 5.1 \%$ & $12.2 \pm 5.1 \%$ & $12.2 \pm 5.1 \%$ & $12.2 \pm 5.1 \%$ & $12.2 \pm 5.1 \%$ \\
\hline Total Events & 36 & 36 & 36 & 36 & 36 \\
\hline At Risk & 4 & 4 & 3 & 3 & 2 \\
\hline $\begin{array}{c}\text { Surgical } \\
\text { Management }\end{array}$ & $64.0 \pm 4.8 \%$ & $64.0 \pm 4.8 \%$ & $62.6 \pm 4.9 \%$ & $62.6 \pm 4.9 \%$ & $62.6 \pm 4.9 \%$ \\
\hline Total Events & 36 & 36 & 37 & 37 & 37 \\
\hline At Risk & 62 & 47 & 44 & 39 & 35 \\
\hline
\end{tabular}

FIGURE 4. Kaplan-Meier survival curves of patients with type A acute aortic dissection presenting with major brain injury stratified by therapeutic management.

unselected patients with TA-AAD presenting with brain injury, irrespective of therapeutic management, with the short- and mid-term outcomes stratified by the presence and type of cerebral damage, in contrast to most previous studies, which assessed only surgical cohorts.

\section{CONCLUSIONS}

Our data have shown that nearly 1 of 10 TA-AAD cases are complicated by major brain injury at the onset of dissection, resulting in a two- or threefold greater risk of mortality, depending on the presence of CVA or coma. The observation that, compared with medical therapy, surgery appeared to be associated with better early and late outcomes and that brain injury reversal occurred in $84.3 \%$ of patients with CVA and $78.8 \%$ of those with coma, suggests that brain injury, per se, should not contraindicate surgery, especially if patients do not present with signs of neurologic devastation. In the present high-risk subcohort of patients with TA-AAD, hypotension/shock/tamponade and renal failure were also confirmed as strong predictors of short- and long-term mortality. Our data suggest that in patients with TA-AAD and neurologic injury, intervention should always be considered.

\section{References}

1. Svensson LG, Kouchoukos NT, Miller DC, Bavaria JE, Coselli JS, Curi MA, et al. Society of Thoracic Surgeons' Endovascular Surgery
Task Force. Expert consensus document on the treatment of descending thoracic aortic disease using endovascular stent-grafts. Ann Thorac Surg. 2008; 85:S1-41.

2. Hiratzka LF, Bakris GL, Beckman JA, Bersin RM, Carr VF, Casey DE Jr, et al. American College of Cardiology Foundation/American Heart Association Task Force on Practice Guidelines; American Association for Thoracic Surgery; American College of Radiology; American Stroke Association; Society of Cardiovascular Anesthesiologists; Society for Cardiovascular Angiography and Interventions; Society of Interventional Radiology; Society of Thoracic Surgeons; Society for Vascular Medicine. 2010 ACCF/AHA/ AATS/ACR/ASA/SCA/SCAI/SIR/STS/SVM guidelines for the diagnosis and management of patients with thoracic aortic disease: a report of the American College of Cardiology Foundation/American Heart Association Task Force on Practice Guidelines, American Association for Thoracic Surgery, American College of Radiology, American Stroke Association, Society of Cardiovascular Anesthesiologists, Society for Cardiovascular Angiography and Interventions, Society of Interventional Radiology, Society of Thoracic Surgeons, and Society for Vascular Medicine. J Am Coll Cardiol. 2010;55: e27-129.

3. Cambria RP, Brewster DC, Gertler J, Moncure AC, Gusberg R, Tilson MD, et al. Vascular complications associated with spontaneous aortic dissection. $J$ Vasc Surg. 1988;7:199-209.

4. Estrera AL, Garami Z, Miller CC, Porat EE, Achouh PE, Dhareshwar J, et al. Acute type A aortic dissection complicated by stroke: can immediate repair be performed safely? J Thorac Cardiovasc Surg. 2006;132:1404-8.

5. Pocar M, Passolunghi D, Moneta A, Mattioli R, Donatelli F. Coma might not preclude emergency operation in acute aortic dissection. Ann Thorac Surg. 2006;81: 1348-51.

6. Tsukube T, Hayashi T, Kawahira T, Haraguchi T, Matsukawa R, Kozawa S, et al. Neurological outcomes after immediate aortic repair for acute type A aortic dissection complicated by coma. Circulation. 2011;124:S163-7.

7. Tanaka H, Okada K, Yamashita T, Morimoto Y, Kawanishi Y, Okita Y. Surgical results of acute aortic dissection complicated with cerebral malperfusion. Ann Thorac Surg. 2005;80:72-6. 
8. Piccione W, Hamilton IN, Najafi H. Intentional delayed repair of acute dissection of the ascending aorta complicated by stroke. J Thorac Cardiovasc Surg. 1995; 109:807-8.

9. Hagan PG, Nienaber CA, Isselbacher EM, Bruckman D, Karavite DJ, Russman PL, et al. The International Registry of Acute Aortic Dissection (IRAD): new insights into an old disease. JAMA. 2000;283:897-903.

10. Mehta RH, Suzuki T, Hagan PG, Bossone E, Gilon D, Llovet A, et al. International Registry of Acute Aortic Dissection (IRAD) Investigators. Predicting death in patients with acute type A aortic dissection. Circulation. 2002;105:200-6.

11. Tsai TT, Bossone E, Isselbacher EM, Nienaber CA, Evangelista A, Fang J, et al. International Registry of Acute Aortic Dissection (IRAD) Investigators. Clinical characteristics of hypotension in patients with acute aortic dissection. Am J Cardiol. 2005;95:48-52.

12. Bonser RS, Ranasinghe AM, Loubani M, Evans JD, Thalji NM, Bachet JE, et al. Evidence, lack of evidence, controversy, and debate in the provision and performance of the surgery of acute type A aortic dissection. J Am Coll Cardiol. 2011; 58:2455-74.
13. Pocar M, Passolunghi D, Moneta A, Donatelli F. Recovery of severe neurologica dysfunction after restoration of cerebral blood flow in acute aortic dissection. Interact Cardiovasc Thorac Surg. 2010;10:839-41.

14. Trimarchi S, Nienaber CA, Rampoldi V, Myrmel T, Suzuki T, Mehta RH, et al. International Registry of Acute Aortic Dissection (IRAD) Investigators. Contemporary results of surgery in acute type A aortic dissection: the International Registry of Acute Aortic Dissection experience. J Thorac Cardiovasc Surg. 2005;129:112-22.

15. Conzelmann LO, Hoffmann I, Blettner M, Kallenbach K, Karck M, Dapunt O, et al. The GERAADA Investigators. Analysis of risk factors for neurological dysfunction in patients with acute aortic dissection type A: data from the German Registry for Acute Aortic Dissection Type A (GERAADA). Eur J Cardiothorac Surg. 2012;42:557-65.

16. Tsai TT, Evangelista A, Nienaber CA, Trimarchi S, Sechtem U, Fattori R, et al International Registry of Acute Aortic Dissection (IRAD) Investigators. Longterm survival in patients presenting with type A acute aortic dissection: insight from the International Registry of Acute Aortic Dissection (IRAD). Circulation 2006;114:I350-6. 
TABLE E1. Independent predictors of follow-up mortality in all patients with type A acute dissection with preoperative brain injury (CVA or coma)

\begin{tabular}{lllr}
\hline \multicolumn{1}{c}{ Variable } & HR & $\mathbf{9 5 \% \text { CI }}$ & $\boldsymbol{P}$ value \\
\hline Hypotension/shock/tamponade & 6.24 & $2.71-14.35$ & $<.001$ \\
Renal failure & 1.97 & $1.03-3.77$ & .041 \\
Surgical management & 0.08 & $0.04-0.20$ & $<.001$ \\
Female gender & 0.69 & $0.36-1.31$ & .253 \\
Age $\geq 70$ y & 1.43 & $0.64-3.17$ & .386 \\
Abnormal ECG findings & 1.63 & $0.80-3.35$ & .180 \\
Abrupt onset of pain & 1.65 & $0.66-4.16$ & .286 \\
History of atherosclerosis & 1.31 & $0.68-2.54$ & .425 \\
Previous cardiac surgery & 0.42 & $0.13-1.33$ & .138 \\
\hline
\end{tabular}

CVA, Cerebrovascular accident; $H R$, hazard ratio; $C I$, confidence interval; $E C G$, electrocardiographic. 